\title{
Comportamiento inicial de especies nativas del Chaco Occidental en plantaciones en zonas de pastizales de altura de las Sierras de Córdoba, Argentina*
}

\author{
Initial performance of Western Chaco native species in high elevation grassland \\ plantations in the Córdoba Range, Argentina
}

\author{
G. VERZINO ${ }^{1}$, J. JOSEAU ${ }^{1}$, M. DEL P. DIAZ ${ }^{2}$, M. DORADO $^{1}$
}

${ }^{1}$ Silvicultura, Facultad de Ciencias Agropecuarias, Universidad Nacional de Córdoba, C.C.509, 5000, Córdoba, Argentina.

${ }^{2}$ Estadística y Biometría, Facultad de Ciencias Agropecuarias, Universidad Nacional de Córdoba, C.C.509, 5000 Córdoba, Argentina.

\section{SUMMARY}

\begin{abstract}
In some sites in the Sierras de Córdoba, the environmental impact of human activity is of such magnitude that natural resource restoration requires afforestation or reforestation measures using native species. The objectives of this study were to evaluate the performance of six Western Chaco native forest species in experimental plantations in high-elevation grasslands in the Córdoba Range, and to identify the best species for the area. Survival and plant height were evaluated in relation to aspect, relative position on the hillside and planting time, in eight experimental plantations which included the following species: Lithraea ternifolia (Gillies) Barkley, Schinopsis haenkeana Engler, Celtis tala Gill. Ex Planchon, Schinus areira L., Prosopis alba Griseb and Prosopis nigra (Griseb) Hieron. The plants' general situation in terms of spatial relationships with other vegetation on the site was also studied. The environmental conditions of the area were adverse for the species' survival and height growth, with the exception of L. ternifolia. The plants showed better survival and general condition when protection was provided by existing vegetation. The most appropriate planting time was spring. Northerly and westerly aspects were most conducive to the species' survival, while the highest height growth was registered for the species Lithraea ternifolia, in the southerly aspect.
\end{abstract}

Key words: reforestation, Western Chaco, mountain watershed, survival, growth.

\section{RESUMEN}

La degradación ambiental por acción antrópica es de tal magnitud, en algunos sitios de las Sierras de Córdoba, que su recuperación requiere de la intervención humana a través de medidas que ayuden a detener y/o revertir el proceso de deterioro. Entre estas medidas se destacan la forestación y la reforestación con especies nativas. Los objetivos del presente trabajo fueron evaluar el comportamiento de 6 especies forestales nativas del Chaco Occidental, en plantaciones experimentales en zonas de pastizales de altura de las Sierras de Córdoba e identificar la especie de mejor comportamiento. En ocho plantaciones se estudiaron la sobrevivencia y altura del eje principal en función de la exposición, la posición en la ladera y la época de plantación de Lithraea ternifolia (Gillies) Barkley, "molle de beber"; Schinopsis haenkeana Engler, "quebracho colorado serrano, horco quebracho"; Celtis tala Gill. Ex Planchon, "tala"; Schinus areira L., "aguaribay, pimentero"; Prosopis alba Griseb, "algarrobo blanco", y Prosopis nigra (Griseb) Hieron, "algarrobo negro". También se analizó el estado general de los plantines en relación a la vegetación acompañante. Las condiciones ambientales de este piso fitogeográfico fueron desventajosas, manifestándose baja sobrevivencia y reducción en la altura promedio luego de varios años de plantación, con excepción de

* El presente proyecto fue ejecutado con fondos provistos por IDRC, Canadá, y la Secretaría de Ciencia y Técnica de la Universidad Nacional de Córdoba. 
BOSQUE 25(1): 53-67, 2004

Comportamiento inicial de especies nativas del Chaco Occidental en plantaciones en zonas de pastizales de altura...

L. ternifolia. Las plantas presentaron mejor sobrevivencia y estado general bajo la protección de los pastos acompañantes, o en el borde de los mismos. La mejor época de plantación fue la primavera y las exposiciones más propicias para el arraigo de las plantas fueron la norte y oeste. En cambio, el mayor crecimiento promedio en altura se produjo en L. ternifolia en la exposición sur.

Palabras claves: reforestación, Chaco Occidental, cuencas de montaña, sobrevivencia, crecimiento.

\section{INTRODUCCION}

Las Sierras de Córdoba presentan una diversidad de ambientes que dan lugar a pisos de vegetación cuya existencia y distribución están condicionadas, principalmente, por la constitución geológica, la altitud y la exposición u orientación geográfica (1). El Bosque Serrano comprende el piso de vegetación más destacado, extendiéndose entre los 500 y los $1.300 \mathrm{~m}$ de altura (1). Por su estratégica localización, en las partes bajas de las cabeceras de las cuencas de captación, esta formación cumple una importante función protectora, influyendo en gran medida en el aporte de agua a los valles donde se localizan los asentamientos humanos. No menos importante en la regulación de las cuencas hídricas es la vegetación de los pisos superiores, el romerillar o matorral serrano y los pastizales de altura, donde tienen origen los cursos de agua que bajan hacia los valles. Tanto unos como otros son objeto de un intenso proceso de degradación por la acción antrópica. La degradación es, en algunos sitios, de tal magnitud que ha conducido a la reducción del potencial biológico del suelo, de manera que se hace extremadamente difícil la recuperación de la vegetación original mediante regeneración natural de las especies. Es indispensable, entonces, la intervención humana a través de medidas que ayuden a detener y/o revertir el proceso de deterioro. Entre estas medidas, la forestación y la reforestación constituyen alternativas muy eficaces (2).

Para llevar adelante acciones de forestación se requiere, previamente, determinar las especies más adecuadas a utilizar. En los pisos superiores, propios del pastizal, se han empleado con éxito diversas coníferas, especialmente pinos, pero no hay, hasta el momento, mayor información sobre forestaciones con especies del Bosque Serrano. Los estudios sobre estas especies se circunscriben, principalmente, a la dinámica y estructura de los bosques (3-6) y a la domesticación de algunas especies para su producción en vivero (7-9), sien- do escasos los antecedentes sobre plantaciones a campo (10).

El uso de árboles de la flora nativa para restaurar tierras degradadas en áreas de pastizales puede ser una opción interesante, considerando la disponibilidad de semillas y la posibilidad de recuperar zonas que anteriormente estaban ocupadas por bosques y se perdieron por causas diversas (incendios, sobrepastoreo, talas excesivas, etc.).

Entre las especies nativas la más representativa es el molle de beber, Lithraea ternifolia (Gillies) Barkley, que junto con el coco, Fagara coco (Gill.) Engler, llegan hasta las mayores alturas de este piso. El otro elemento principal del bosque es el horco quebracho o quebracho serrano, Schinopsis haenkeana Engler, que ocupa solamente la faja inferior del piso forestal, alcanzando en algunas áreas los 1.000 o $1.100 \mathrm{~m}$ de elevación. De los árboles de la llanura que ascienden parcialmente los faldeos inferiores o que penetran por los valles, cañadones y quebradas hasta $1.000 \mathrm{~m}$ de elevación, se destacan los algarrobos blancos, Prosopis alba Griseb. y Prosopis chilensis (Mol.) Stuntz, algarrobos negros, Prosopis nigra (Griseb) Hieron y $P$. flexuosa D.C., el espinillo, Acacia caven (Mol.) Molina, y el tala, Celtis tala Gill. Ex Planchon (1).

Si bien se carece de información sobre plantaciones de molle y horco quebracho, se conoce que el primero tiende a ocupar naturalmente los sitios más frescos y húmedos, con preferencia en las laderas expuestas al sur $(1,3)$, mientras que el segundo "ocupa, en Argentina, vertientes con orientación norte y oeste preferentemente, más secas y cálidas, donde puede constituir comunidades puras de densidad variable" (1). También prospera en laderas orientales, como las de las Sierras Norte y Orcosuni, el cordón sedimentario Pajarillo-Copacabana-Masa, y en valles longitudinales de las Sierras Chicas (El Diquecito, en el Depto. Santa María), mostrando la influencia de otros factores, además de los termopluviométricos, en el desarrollo y dispersión de la especie. En Bolivia, horco quebracho crece en 
BOSQUE 25(1): 53-67, 2004

Comportamiento inicial de especies nativas del Chaco Occidental en plantaciones en zonas de pastizales de altura...

laderas secas y pedregosas, con fuertes pendientes, trepando hasta $2.700 \mathrm{~m}$ s.n.m. (11).

En cuanto a plantaciones con algarrobos, si bien existen numerosas experiencias, éstas provienen mayormente de zonas llanas $(12,13)$, siendo necesario conocer su aptitud para uso en reforestaciones en zonas de sierras. Sobre la tala, una de las especies de más amplia distribución natural en Argentina (14), no se hallaron antecedentes de plantaciones, salvo con fines ornamentales y de protección de animales $(15,16)$.

Por su parte, aguaribay, Schinus areira L, nativa del centro y sur de Sudamérica, ha mostrado una asombrosa adaptación en introducciones espontáneas. Brack Egg (17) menciona plantaciones exitosas de aguaribay, en zonas de sierra de Perú, con fines de protección ambiental, ornamental o de producción de leña, lo que induciría a esperar buena respuesta en similares experiencias en las Sierras de Córdoba.

Los factores que inciden en la distribución de la vegetación natural en pisos, en zonas de sierras, tales como temperatura, precipitaciones, vientos, composición geológica y suelo (1), son a su vez determinantes del éxito o el fracaso de una plantación en un sitio dado. La exposición y la ubicación de las plantas en la ladera inciden igualmente en la sobrevivencia y el posterior crecimiento $(18,19)$.

Entre las variables silviculturales, la época de plantación y la posición relativa del plantín con respecto a la vegetación existente pueden afectar el comportamiento inicial de las plantas. Así, por ejemplo, el establecimiento de plantaciones de coníferas en las Sierras de Córdoba es más exitoso si se realiza en el otoño, época de menor déficit hídrico (25). Por su parte, estudios recientes indican que la instalación de ciertas especies de la región chaqueña occidental requiere de la protección de otras especies $(20,21)$.

Los objetivos del presente trabajo son los siguientes: a) evaluar el comportamiento inicial de 6 especies forestales nativas del Chaco Occidental, en plantaciones experimentales en zonas de pastizales de altura de las Sierras de Córdoba, en función de la época de plantación, la exposición, la ubicación en la ladera y la posición relativa con respecto a la vegetación acompañante, y b) identificar la/s especie/s de mejor desempeño, en términos de sobrevivencia y crecimiento en altura, en las condiciones del ensayo.

\section{MATERIAL Y METODOS}

El ensayo se efectuó en la cuenca alta del arroyo Vaquerías, dentro de la Reserva Natural Vaquerías, propiedad de la Universidad Nacional de Córdoba, sobre la vertiente occidental del cordón de las Sierras Chicas, a $31^{\circ} 06^{\prime}$ latitud Sur, 64 26' longitud Oeste, entre 1.150 y 1.250 m s.n.m. Las precipitaciones registradas en zonas aledañas (La Falda) promedian los $700 \mathrm{~mm}$ anuales, concentrados mayormente en primavera y verano. La temperatura máxima media de enero es $27^{\circ} \mathrm{C}$ y la mínima media de julio es $4^{\circ} \mathrm{C}$, con máxima y mínima absolutas de $38^{\circ} \mathrm{C}$ y $-8,8^{\circ} \mathrm{C}$ respectivamente.

Las especies seleccionadas fueron molle, $L$. ternifolia, horco quebracho, $S$. haenkeana, tala, $C$. tala, aguaribay, S. areira y algarrobos, $P$. alba y $P$. nigra. El ensayo consistió en ocho plantaciones experimentales dispuestas en lotes de $52 \mathrm{~m}$ x 12 $\mathrm{m}$, cercados con alambre tejido. A fin de estudiar el efecto de la exposición, la posición en la ladera y la época de plantación, en marzo de 1995 se instalaron cuatro plantaciones en la parte alta de laderas orientadas, respectivamente, hacia los cuatro puntos cardinales (denominadas, de aquí en adelante, plantaciones en ladera alta). En noviembre de 1997 se efectuaron las cuatro restantes, en la parte baja de las mismas laderas (de aquí en adelante, plantaciones en ladera baja). En esa misma fecha se repusieron las plantas muertas en ladera alta (reposición de fallas). Esto permitió, luego, comparar las dos épocas de plantación (otoño vs primavera) y la posición en la ladera (ladera alta vs ladera baja) en términos de sobrevivencia y altura de las plantas. Por falta de plantines no se efectuó reposición de $P$. nigra, salvo 5 plantas en exposición sur y una en exposición oeste. Dado el bajo crecimiento de las plantas, la reposición de fallas, realizada 32 meses después de la plantación, no sufrió competencia por parte de las plantas ya instaladas, por lo que pudo considerarse como una nueva plantación.

Tanto en ladera alta como ladera baja se instaló un ensayo bajo un diseño en bloques completamente aleatorizados con estructura de tratamientos en parcelas divididas, siendo las cuatro exposiciones (norte, sur, oeste y este) los tratamientos principales y las seis especies los secundarios. El diseño constaba de 4 plantas por unidad experimental en 5 repeticiones de modo tal que resultaron 120 
BOSQUE 25(1): 53-67, 2004

Comportamiento inicial de especies nativas del Chaco Occidental en plantaciones en zonas de pastizales de altura...

plantas por plantación (20 ejemplares/especie x 6 especies), 240 plantas por exposición y 960 plantas en total.

Los plantines se produjeron en el vivero forestal de Silvicultura (FCA, UNC), situado en la ciudad de Córdoba. Se usaron envases de polietileno cristal de $560 \mathrm{~cm}^{3}$, con una mezcla de tierra y arena gruesa. Se protegieron con media sombra y se regaron convenientemente. A campo, las plantas de 6-12 meses (altura de 15-40 cm según especie) se dispusieron en marco real, a $2 \mathrm{~m} \times 2 \mathrm{~m}$. Se efectuó un riego de plantación y control periódico de hormigas.

Registros a campo: Sobre cada planta se midieron las siguientes variables:

\section{Ladera alta:}

$\checkmark$ Plantación marzo/1995 (otoño):

sobrevivencia a los 7 y 61 meses de la plantación, altura del eje principal a los 0, 7, 19, 24, 32, $37 \mathrm{y}$ 61 meses de la plantación,

condición del plantín a los 61 meses de la plantación,

posición relativa del plantín con respecto a la vegetación existente a los 61 meses de la plantación.

$\checkmark$ Reposición de fallas noviembre/1997 (primavera):

sobrevivencia a los 5 y 29 meses de la plantación, altura del eje principal a los 0, 5 y 29 meses de la plantación;

\section{Ladera baja}

$\checkmark$ Plantación noviembre/1997 (primavera): sobrevivencia a los 29 meses de la plantación, altura del eje principal a los 0, 5 y 29 meses de la plantación,

condición del plantín a los 29 meses de la plantación,

posición relativa del plantín con respecto a la vegetación existente a los 29 meses de la plantación.

Dado que la reposición en ladera alta y la plantación en ladera baja se realizaron 36 meses después de la iniciación del ensayo en ladera alta, la última medición, que en ladera alta se efectuó a los 61 meses de la plantación, coincidió con los 29 meses de la reposición y de la plantación en ladera baja.
La variable condición del plantín fue utilizada para reflejar el estado general de los mismos a través de una escala cualitativa. Esta considera la altura del eje principal, el número y estado de las hojas, el color y el estado sanitario. Se determinaron las siguientes categorías en escala ordinal: 1. malo, 2. regular, 3. bueno y 4. muy bueno. Por ejemplo, la categoría 4 es otorgada a un plantín alto, vigoroso, con abundante cantidad de hojas, de color verde y buena sanidad, mientras que se categorizó 1 a un plantín débil, con muy pocas hojas, o sin ellas, cloróticas o enfermas.

La variable posición relativa del plantín con respecto a la vegetación existente se generó para evaluar el efecto de la vegetación acompañante sobre la sobrevivencia de los plantines. Se determinaron 3 categorías: 1. bajo cobertura, 2. ecotono (en el borde de la cobertura) y 3 . fuera ( $\sin$ cobertura).

Análisis de la información. Se efectuaron análisis descriptivos y gráficos con el objeto de visualizar el comportamiento general de las variables.

Sobrevivencia. La variable sobrevivencia, cuyo valor dado fue 1 , para plantas vivas, y 0 , para plantas muertas, fue analizada respetando su naturaleza dicotómica, es decir no continua. En este caso los modelos normales clásicos no pueden ser aplicados, por lo que para evaluar los efectos de los tratamientos (combinaciones de los niveles de los factores) se ajustaron modelos especiales, como los Modelos Lineales Generalizados (MLG) (22), que permitieron obtener estimaciones confiables de las proporciones esperadas de éxito (sobrevivencia) de las especies. Todos los modelos fueron ajustados en GLIM (Generalized Linear Iterative Models) (23).

El efecto de la época de plantación sobre la sobrevivencia se analizó comparando la plantación otoñal (marzo/95) y la reposición de fallas efectuada en primavera (nov/97), en ladera alta, a los 7 y 5 meses de la implantación, respectivamente. El efecto de la ubicación en la ladera (ladera alta vs ladera baja) se evaluó al comparar la sobrevivencia de la reposición de fallas en ladera alta con la plantación en ladera baja (efectuadas ambas en nov/97), luego de 5 meses de plantadas.

La posible asociación entre sobrevivencia y condición del plantín, así como entre sobrevivencia y posición relativa con respecto a la vegetación 
existente, fue evaluada ajustando MLG's para respuesta categórica de tipo ordinal, que se basan en las probabilidades acumuladas (23).

Altura del eje principal. Fueron ajustados modelos gaussianos con componente sistemático correspondiente a un modelo de análisis de la varianza con una restricción a la aleatorización y estructura factorial de tratamientos, siendo los factores las exposiciones y las especies Dada la dependencia significativa entre las respuestas de altura a lo largo del tiempo, los modelos ajustados incorporaron información de un proceso autorregresivo de segundo orden analizando los perfiles de altura para cada una de las especies siguiendo la metodología clásica de los MLG (22).

El efecto de la época de plantación sobre la altura de las plantas se analizó comparando la plantación otoñal (marzo/95) y la reposición de fallas efectuada en primavera (nov/97), en ladera alta, a los 7 y 5 meses de la implantación, respectivamente. El efecto de la ubicación en la ladera (ladera alta vs ladera baja) se evaluó sobre tres de las seis especies del ensayo: horco quebracho, molle y algarrobo blanco (que hasta el momento tenían buena sobrevivencia), plantadas simultáneamente en ladera alta y baja (en nov/97), luego de 5 meses de la plantación.

Mientras algunos factores, como exposición y posición con respecto a la vegetación acompañante, se estudiaron temporalmente en períodos de años (5 años en ladera alta y 2,5 años en ladera baja), otros factores, como época de plantación y ubicación en la ladera solamente pudieron analizarse en un intervalo menor de meses desde la plantación, debido a la ausencia de balanceamiento en la estructura de datos, causado por la alta mortalidad en algunas de las especies.

\section{RESULTADOS Y DISCUSION}

\section{SOBREVIVENCIA}

En función de la época de plantación. En términos generales, la sobrevivencia al cabo de medio año de la plantación fue mayor $(\mathrm{p}<0,0001)$ en la plantación primaveral. Algunas especies tuvieron valores promedio de sobrevivencia muy bajos en plantación otoñal, hasta hacerse cero en el caso de horco quebracho. Este comportamiento no depen- dió de la exposición a la que estuviera sometida la especie $(p=0,2110)$, indicando ausencia de interacción época de plantación-exposición. Por su parte, las exposiciones difirieron entre sí ( $\mathrm{p}=0,047)$, siendo la Este la más adversa tanto en plantación otoñal como primaveral.

Se observó interacción entre la época de plantación y la especie $(\mathrm{p}=0,0012)$. En la plantación otoñal los algarrobos tuvieron buena respuesta en todas las exposiciones, molle fue mejor en la exposicion Sur y horco quebracho no sobrevivió. En la plantación de primavera, el desbalanceamiento de datos dificultó el análisis de contrastes específicos que permitieran indagar la naturaleza de la interacción. El promedio de sobrevivencia de las especies estuvo entre el $93 \%$ y el $99 \%$, no existiendo, por lo tanto, diferencias significativas entre ellas $(\mathrm{p}=0,1141)$ (figura 1$)$.

En función de la ubicación en la ladera. El análisis de la sobrevivencia en ladera alta y en ladera baja, luego de cinco meses de la plantación primaveral, indica que en ambas existe interacción de los factores exposición y especie ( $\mathrm{p}=0,05$ y $\mathrm{p}=0,001$, respectivamente). En ladera alta, la exposición Este resulta la más desfavorable para todas las especies, particularmente para el tala. En ladera baja, también esta exposición fue la de menor sobrevivencia, pero solamente para el tala y el algarrobo negro (figura 2).

En función de la exposición. Tanto en ladera alta como ladera baja se detectó interacción significativa entre los factores exposición y especie $(p=0,00313$ y $\mathrm{p}<0,0001$, respectivamente) debido a que las especies no se comportan de la misma forma en todas las exposiciones.

No obstante, la exposición Norte fue, en términos generales, la de mejor desempeño $(\mathrm{p}<0,0001)$ al cabo de 61 meses de la plantación. Así, por ejemplo, en ladera alta, el molle mostró una sobrevivencia significativamente mayor $(\mathrm{p}<0,00001)$ que las demás especies. El aguaribay y los algarrobos negro y blanco, de comportamiento similar $(\mathrm{p}=0,8237)$, mostraron mayor tendencia a sobrevivir (30-35\%) en la exposición Norte ( $\mathrm{p}=0,0911)$, inversamente a lo que ocurrió con horco quebracho y tala (ambos semejantes, $\mathrm{p}=0,875$ ), siendo esta última la especie de menor sobrevivencia (figura 3). 
BOSQUE 25(1): 53-67, 2004

Comportamiento inicial de especies nativas del Chaco Occidental en plantaciones en zonas de pastizales de altura...

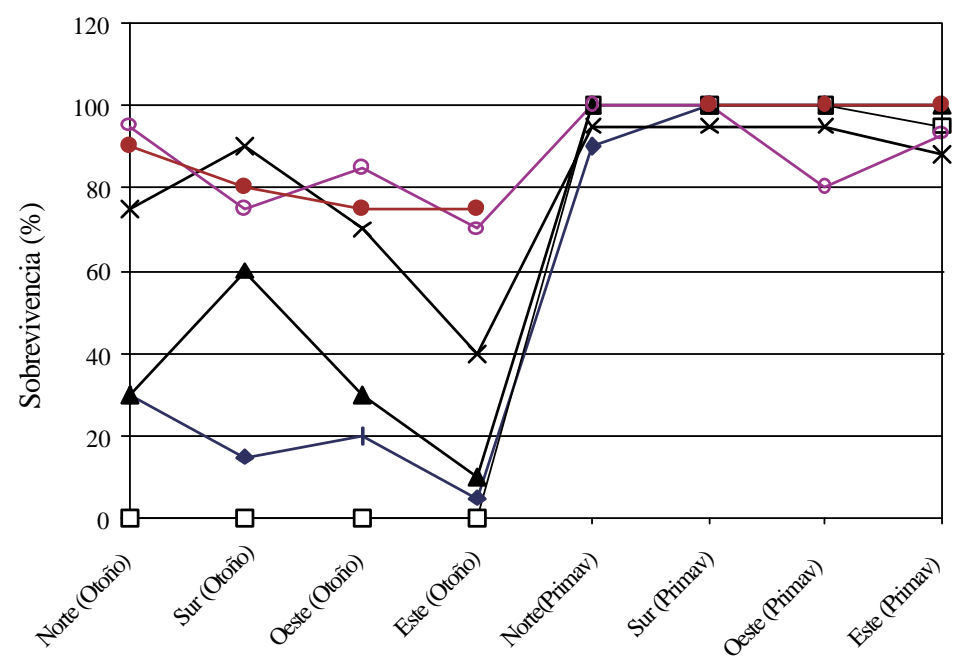

Exposición/Epoca de plantación

\begin{tabular}{|ll|}
$\longrightarrow$ Aguaribay & $-\square$ - Horco quebracho \\
$\rightarrow$ Molle & $\rightarrow-$ Tala \\
- Algarrobo blanco & - Algarrobo negro \\
\hline
\end{tabular}

Figura 1. Sobrevivencia (\%) en las cuatro exposiciones, al cabo de 7 y 5 meses de la plantación otoñal y primaveral, respectivamente, en ladera alta. Percentage survival rate by aspect, 7-5 months after fall/spring planting time, respectively. High hillside position.

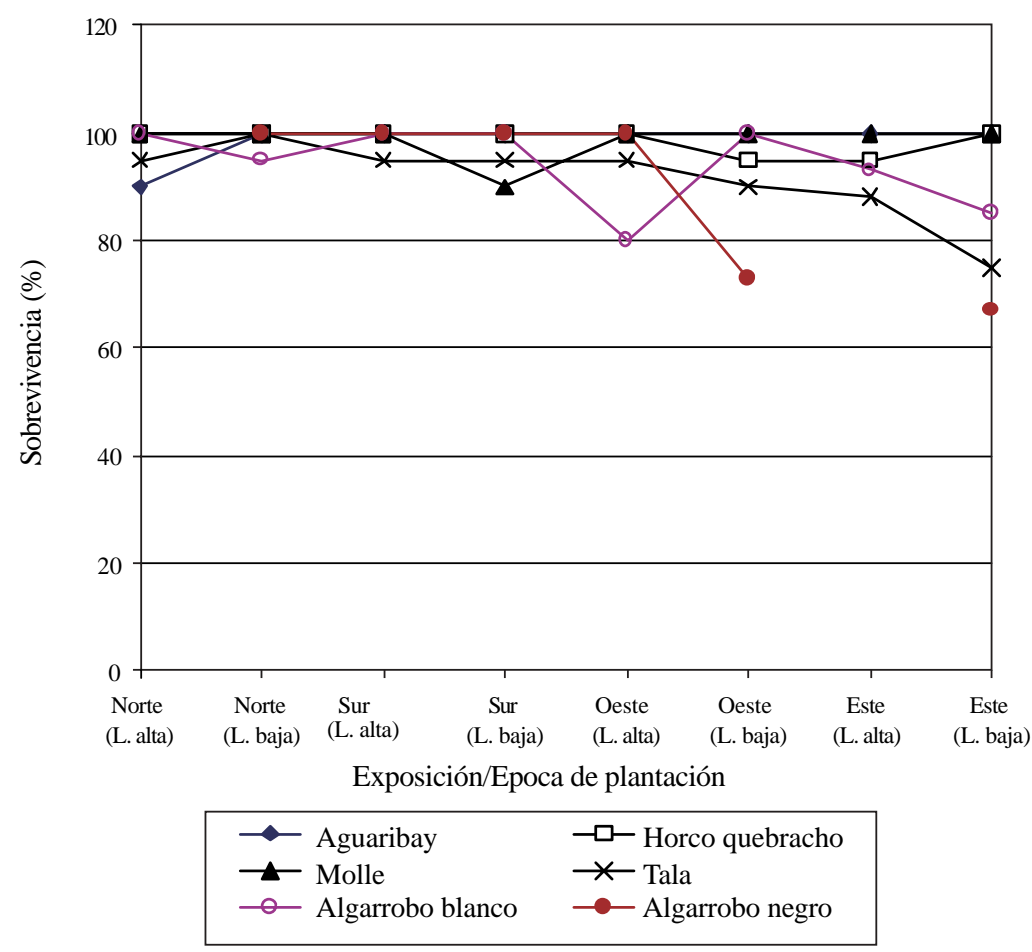

Figura 2. Sobrevivencia (\%) al cabo de 5 meses de la plantación primaveral, en función de la exposición y la ubicación en la ladera.

Percentage survival rate five months after spring planting, with regard to aspect and hillside position. 


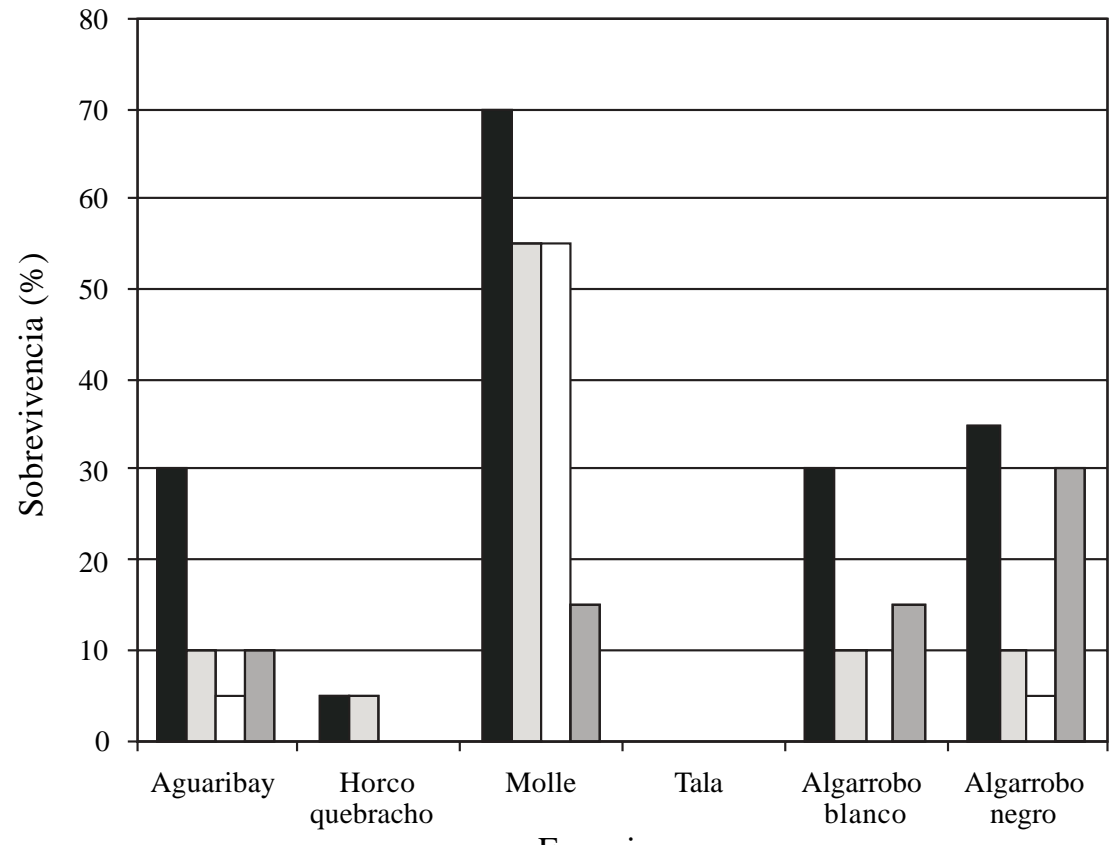

Especie

$\square$ Norte $\square$ Sur $\square$ Oeste $\square$ Este

Figura 3. Sobrevivencia (\%) de las seis especies estudiadas, discriminada por exposición, a los 61 meses de la plantación en ladera alta.

Percentage survival rate of the six species, by aspect, 61 months after planting on high hillside.

En ladera baja, a los 29 meses de la plantación, molle también presentó mayor sobrevivencia que el resto de las especies; sin embargo, la exposición más favorable fue la Oeste $(\mathrm{p}=0,1939)$. Solamente en las exposiciones Norte y Este algarrobo blanco se asemejó a molle en cuanto a su alta sobrevivencia. El resto de las especies presentaron alta mortalidad en todas las exposiciones (figura 4).

En función de la vegetación acompañante. En las plantaciones en ladera alta la sobrevivencia general de las especies fue semejante $(\mathrm{p}=0,2499)$ en las categorías bajo cobertura $-1-\mathrm{y}$ ecotono $-2-$, salvo en la exposición Este, donde las plantas tuvieron mayor sobrevivencia bajo cobertura. No obstante, como existe asociación $(\mathrm{p}=0,0114)$ entre especie y categoría, en el análisis por especie es posible observar que, mientras la mayoría de las especies sobrevivieron significativamente bajo cobertura, molle sobrevivió indistintamente bajo cobertura o en el borde (ecotono), salvo en la ex- posición Este, donde prefirió la posición bajo cobertura (figura 5).

En ladera baja, las especies con mayor sobrevivencia (molle, algarrobo blanco y aguaribay) también lo hicieron, en términos generales, bajo cobertura o en el borde (ecotono). No hubo interacción $(\mathrm{p}=0,3857)$ entre los factores exposición y especie dada la ubicación del individuo; sólo molle mostró cierta tendencia a sobrevivir en la exposición Sur, fuera de la protección de la vegetación existente.

Condición de los plantines. En ladera alta, a los 61 meses de la plantación, las especies presentaban condiciones generales diferentes entre sí ( $\mathrm{p}=0,0654)$, destacándose el molle por su condición buena y muy buena, en todas las exposiciones. Se observó, además, que los mejores individuos se hallaron, predominantemente, bajo cobertura o en el borde de la vegetación existente.

En ladera baja, molle también sobresalió por mostrar individuos buenos y muy buenos, pero esta 
BOSQUE 25(1): 53-67, 2004

Comportamiento inicial de especies nativas del Chaco Occidental en plantaciones en zonas de pastizales de altura...

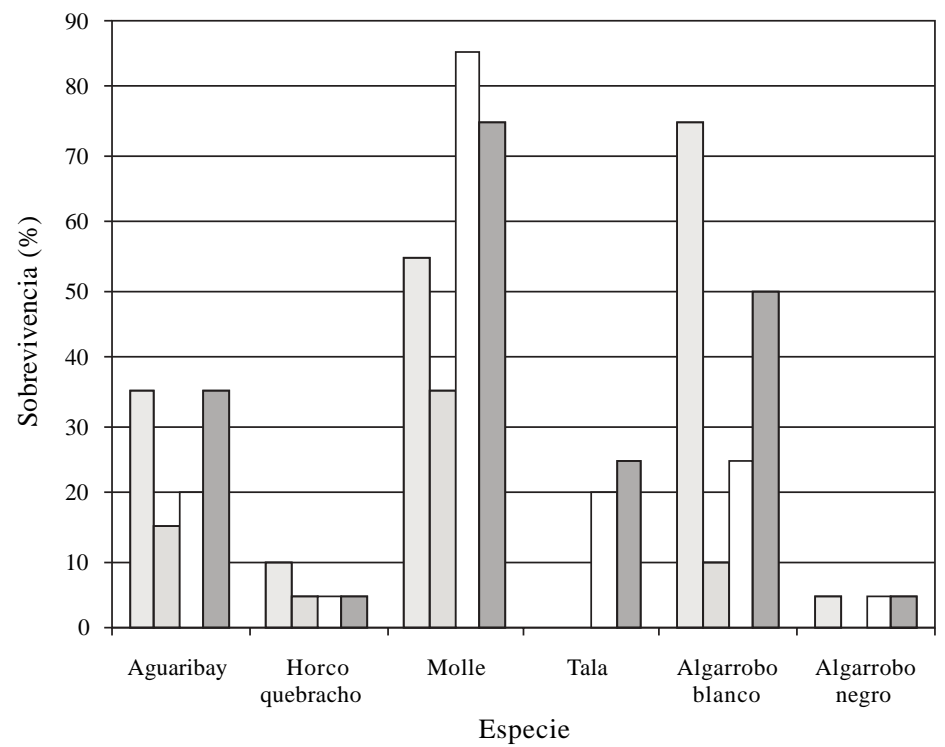

$\square$ Norte $\square$ Sur $\square$ Oeste $\square$ Este

Figura 4. Sobrevivencia (\%) de las seis especies estudiadas, discriminada por exposición, a los 29 meses de la plantación en ladera baja.

Percentage survival rate of the six species, by aspect, 29 months after planting on low hillside.
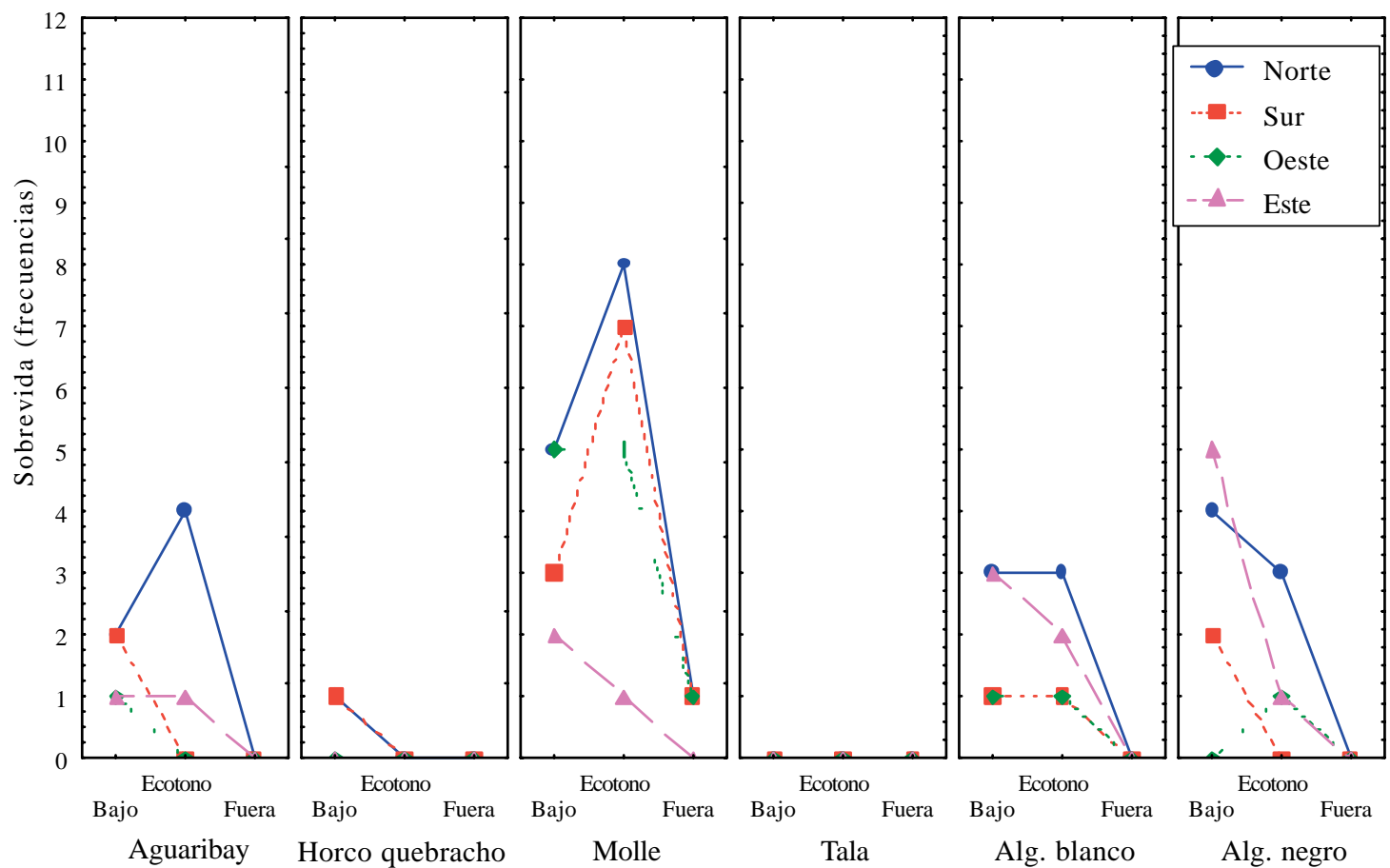

Figura 5. Sobrevivencia de las especies, en frecuencia absoluta -máximo valor: 20-, en las cuatro exposiciones, en función de su posición con respecto a la vegetación acompañante, a los 61 meses de la plantación en ladera alta. Species survival rate related to the plants' spatial relationship with other vegetation, in absolute frequency -maximum value: 20-, by aspect, 61 months after planting on high hillside. 
BOSQUE 25(1): 53-67, 2004 Comportamiento inicial de especies nativas del Chaco Occidental en plantaciones en zonas de pastizales de altura..

vez acompañado por aguaribay, y en todas las exposiciones, con una tendencia a la disminución en exposición Sur (figura 6).

\section{ALTURA DEL EJE PRINCIPAL}

En función de la época de plantación. Al analizar las diferencias de alturas entre el momento de la plantación/reposición y la siguiente medición, efectuada a los 7 y 5 meses respectivamente, todas las especies mostraron diferencias significativas $(\mathrm{p}=0,028)$ entre ambas épocas de plantación. La plantación primaveral resultó la de mejor desempeño y, entre las exposiciones, la Sur fue la más favorable $(\mathrm{p}=0,05)$, mientras que la Este fue la peor. El molle, con buen crecimiento en plantación primaveral, no mostró la misma respuesta en plantación de otoño, a pesar de ser una de las especies menos perjudicadas durante el invierno (cuadro 1).
En función de la ubicación en la ladera. Las tres especies analizadas (horco quebracho, molle y algarrobo blanco) crecen más en ladera alta que baja en la exposición Sur $(p=0,05)$. Algarrobo blanco repite este patrón en el resto de las exposiciones, mientras que molle sólo en la Norte (figuras 7 y 8).

En función de la exposición. La figura 7 muestra la evolución de la altura del eje principal de las seis especies estudiadas en las cuatro exposiciones, desde el momento de la plantación otoñal en ladera alta y hasta la última medición, a los 61 meses. Algunas plantas de horco quebracho y aguaribay rebrotaron de la base luego de haber sido registradas como muertas, en la medición anterior, produciendo entonces curvas de altura atípicas.

En ladera alta la única exposición que mostró diferencia positiva contrastante con el resto fue la exposición Sur $(p=0,0251)$. Las exposiciones Norte y Oeste fueron las de peor respuesta.

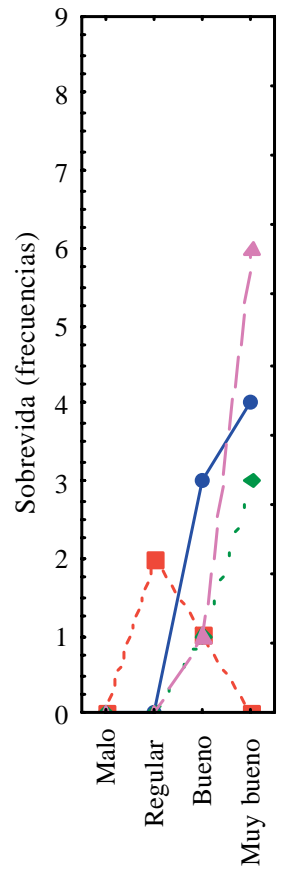

Aguaribay

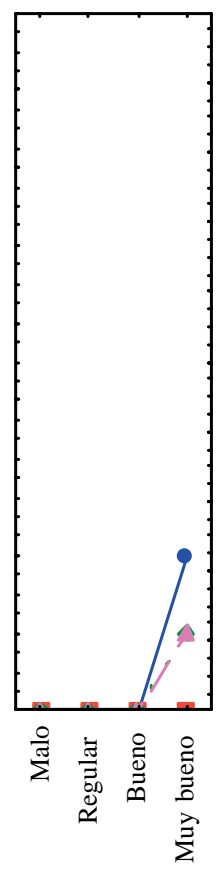

Horco quebracho

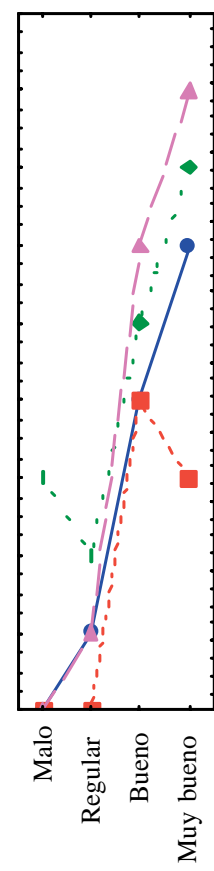

Molle

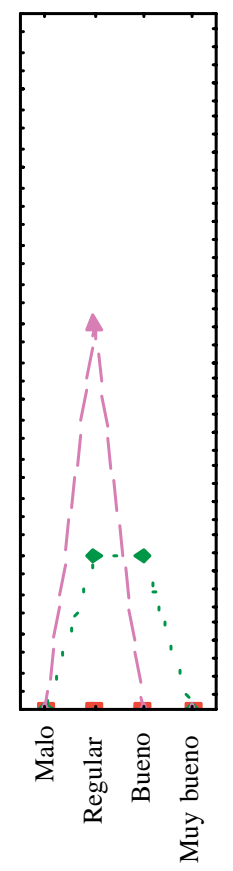

Tala

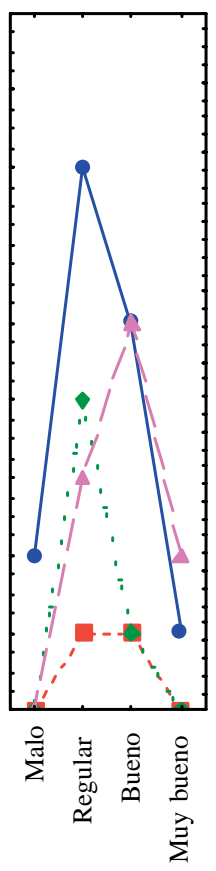

Algarrobo blanco Algarrobo negro

Figura 6. Sobrevivencia de las especies, en frecuencia absoluta -máximo valor: 20-, en las cuatro exposiciones, discriminada según condición de los plantines, a los 29 meses de la plantación en ladera baja.

Species survival rate related to the plants' spatial relationship with other vegetation, in absolute frequency -maximum value: 20-, by aspect, 61 months after planting on high hillside. 
BOSQUE 25(1): 53-67, 2004

Comportamiento inicial de especies nativas del Chaco Occidental en plantaciones en zonas de pastizales de altura...

\section{CUADRO 1}

Diferencias de altura $(\mathrm{cm})$, a los 7 y 5 meses de la plantación otoñal y primaveral respectivamente, en ladera alta. Height differences $(\mathrm{cm})$, after 7-5 months from the fall/spring planting, respectively. High hillside positions.

\begin{tabular}{|l|r|r|r|r|r|r|r|r|}
\hline \multirow{2}{*}{ Especie } & \multicolumn{4}{|c|}{ Plantación otoñal } & \multicolumn{4}{c|}{ Plantación primaveral } \\
\cline { 2 - 8 } & Norte & \multicolumn{1}{|c|}{ Sur } & Oeste & Este & Norte & Sur & Oeste & Este \\
\hline Aguaribay & $-1,0$ & $-4,4$ & $-11,7$ & $-21,8$ & 1,6 & 9,3 & $-4,7$ & $-3,1$ \\
Horco quebracho & $\mathrm{M}$ & $\mathrm{M}$ & $\mathrm{M}$ & $\mathrm{M}$ & 2,6 & 2,3 & $-0,9$ & 0 \\
Molle de beber & 1,2 & 1,0 & 1,4 & $-14,8$ & 7,6 & 10,6 & 3,9 & 7,9 \\
Tala & $-3,4$ & $-3,7$ & $-5,7$ & $-4,1$ & 0,7 & $-0,9$ & $-0,2$ & 1,2 \\
Algarrobo blanco & $-2,9$ & $-10,3$ & $-5,8$ & 3,7 & 8,9 & 6,4 & $-5,3$ & 8,5 \\
Algarrobo negro & $-1,6$ & $-2,4$ & $-6,2$ & $-1,6$ & - & 2,0 & $-0,5$ & - \\
\hline
\end{tabular}

Referencias: Los valores negativos indican reducción de la altura al cabo del período considerado.

M: Todas las plantas se presentaban muertas a los 5 meses de la plantación otoñal.

- : No se plantaron individuos de esta especie.

References: Negative values indicate reduction in height after the measured period.

M: All plants were dead.

-: No plants of this species were planted.
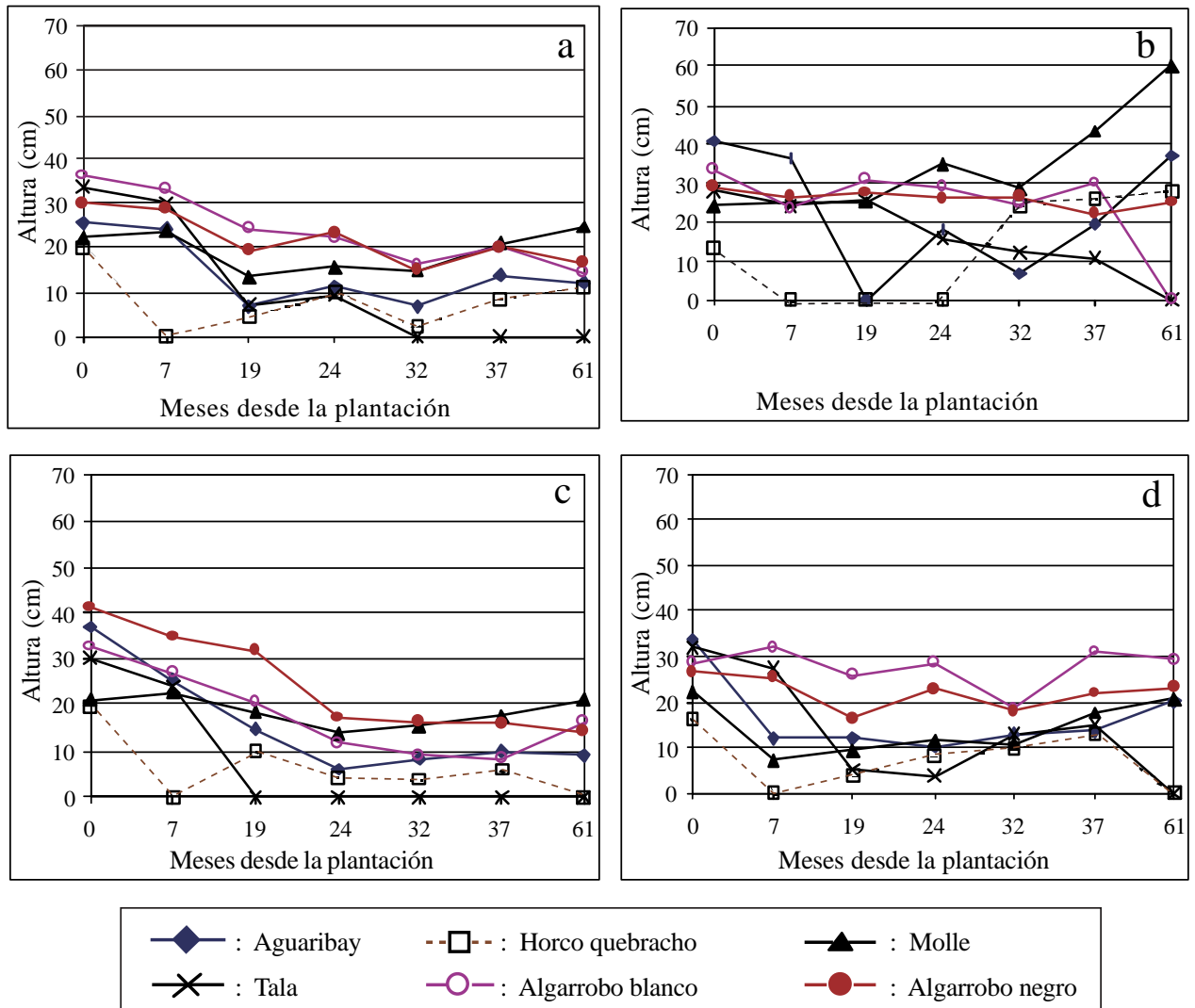

Figura 7. Perfiles de altura del eje principal de todas las especies desde el momento de la plantación otoñal (fecha 0 ) y hasta la última medición (a los 61 meses -5 años-), en ladera alta. Referencias: a) exposición norte; b) exposición sur; c) exposición oeste y d) exposición este.

Profiles for height growth in the six species from planting time in the fall (date 0 ) up to the last measurement (after 61 months), on high hillside location. References: a) northerly aspect, b) southerly aspect, c) westerly aspect, d) easterly aspect. 
En términos generales, las especies también mostraron desempeños distintos $(\mathrm{p}<0,0001)$. No obstante, como no todas las especies sobrevivieron en todas las exposiciones, esas diferencias fueron analizadas sólo en la exposición Sur. Molle se destacó con un crecimiento positivo en altura, seguido por aguaribay. La primera especie alcanzó alturas promedio aproximadamente tres veces superiores a la inicial al cabo de 5 años de plantación $(24,28 \mathrm{~cm}$ el $19 / 3 / 95$ vs $60,27 \mathrm{~cm}$ el $27 / 3 / 00)$ (figura $7 b$ ).

La figura 8 muestra la evolución de la altura del eje principal de las seis especies estudiadas en las cuatro exposiciones, desde el momento de la plantación primaveral en ladera baja y hasta la última medición, a los 29 meses.
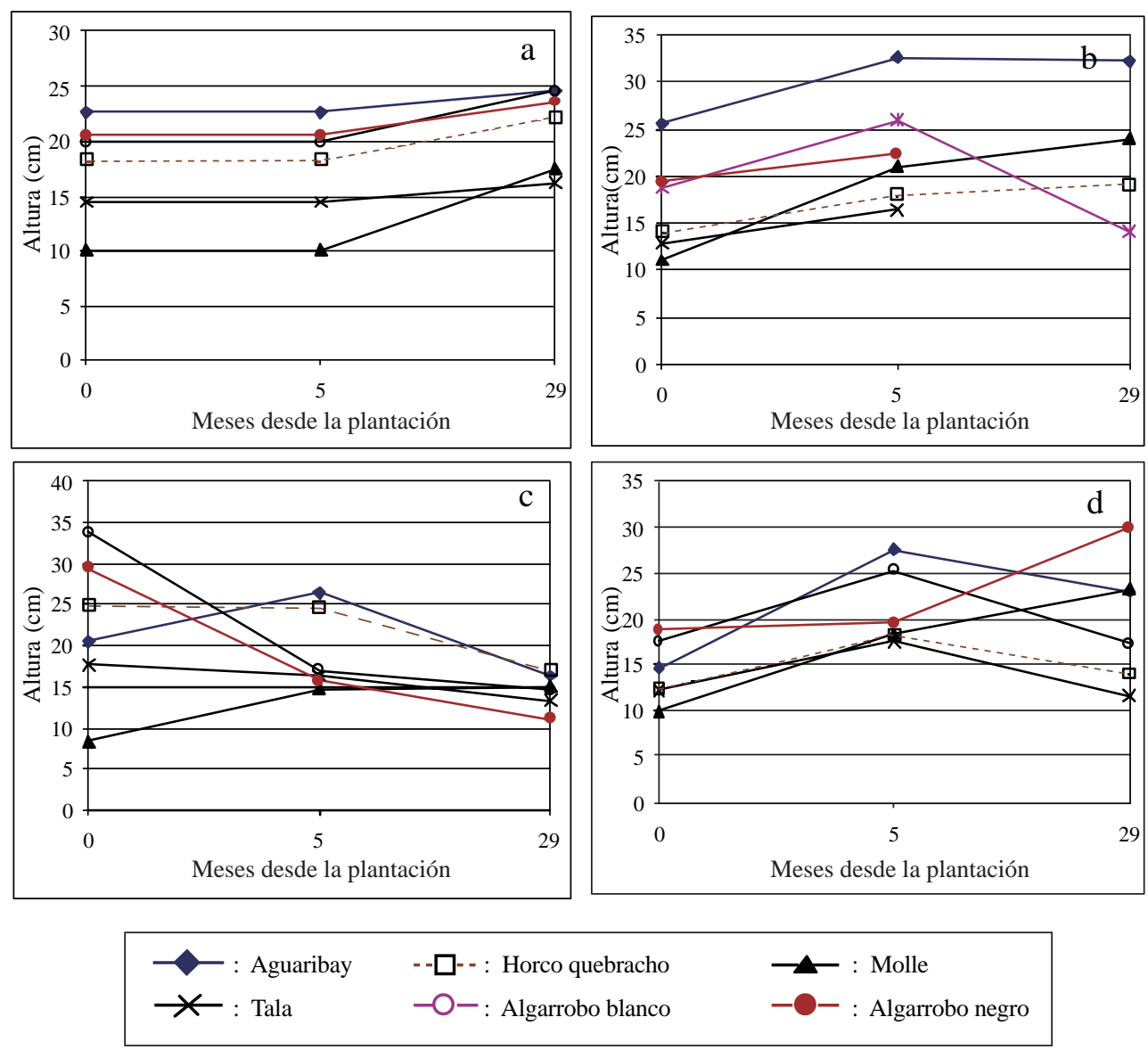

Figura 8. Perfiles de altura del eje principal de todas las especies desde el momento de la plantación primaveral (fecha 0) y hasta la última medición (a los 29 meses). Ladera baja. Referencias: a) exposición norte; b) exposición sur; c) exposición oeste y d) exposición este.

Profiles for height growth in the six species from planting time in the spring (date 0 ) up to the last measurement (29 months afterwards), on low hillside location. References: a) northerly aspect, b) southerly aspect, c) westerly aspect, d) easterly aspect.
En ladera baja, los plantines de la exposición Oeste, que habían presentado la mayor tasa de sobrevivencia, mostraron tendencia a crecer menos que en las exposiciones Este y Sur ( $\mathrm{p}=0,1096)$. Se detectó interacción de los factores exposición y especie, indicando que las especies se comportaron diferencialmente según la exposición. Este comportamiento diferencial está dado por $P$. nigra en la exposición Este, y a pesar de su bajísima altura significativamente mayor $(\mathrm{p}=0,0015)$ que resto de las especies.

De los resultados se desprende que las especomportan diferencialmente según la época de plantación, la ubicación en la ladera, la exposición y la posición de los individuos con respecto a 
la vegetación acompañante. Asimismo, las variables sobrevivencia y altura del eje principal no guardan el mismo patrón de comportamiento en todas las especies, de modo que a mayor sobrevivencia en un sitio dado no siempre corresponde un mayor crecimiento en altura.

El alto nivel de mortandad de todas las especies del ensayo, atribuible a las condiciones climáticas del sitio (bajas temperaturas, alta evapotranspiración) y factores bióticos no controlados (ataque de liebres, hormigas, competencia de malezas), dificultó algunas etapas del análisis estadístico. Sin embargo, los datos disponibles permitieron arribar a conclusiones válidas.

Entre las especies, molle resultó la mejor, en términos de sobrevivencia, altura del eje principal y condición general de los plantines, particularmente en ladera alta. Cabe notar que mientras la sobrevivencia de esta especie al cabo de 5 años fue mayor en exposición Norte $(70 \%)$, el mejor crecimiento en altura se produjo en la exposición Sur. Esta última favoreció, además, una muy buena condición general de los plantines, en concordancia con observaciones efectuadas por diversos autores $(1,3)$ que indican la preferencia natural de molle para ocupar los sitios más frescos y húmedos en las laderas expuestas al Sur.

Aguaribay tuvo un pobre desempeño. Su sobrevivencia fue baja en todas las exposiciones (máxima $38 \%$, en exposición Norte, ladera baja) y su altura no varió significativamente con respecto a la altura de plantación, en promedio, cualquiera fuera la exposición o la posición en la ladera (figuras 8 y 9). Era de esperar que la especie tuviera mejor performance debido a que crece naturalmente en Perú, en estepas espinosas y bosques montanos bajos, trepando hasta los 3.650 m s.n.m.; allí es usada con buenos resultados en sistemas silvopastorales (24). Asimismo, Brack Egg (17) menciona plantaciones exitosas de Schinus areira en zonas de sierra de Perú con fines de protección ambiental, ornamental o de producción de leña.

Horco quebracho y tala se mostraron totalmente inadecuadas para plantaciones en la zona, lo que queda de manifiesto por su bajísima tasa de sobrevivencia al final del ensayo. Si bien horco quebracho crece en Bolivia en laderas secas y pedregosas, con fuertes pendientes, hasta 2.700 m s.n.m. (11), es probable que las condiciones de reclutamiento de la especie en los sitios de dispersión natural sean menos adversas que las del ensa- yo. El tala, por su parte, mostró muy pobre desempeño. Al cabo de cinco años de la plantación otoñal en ladera alta la sobrevivencia fue nula en todas las exposiciones. Siendo el tala una especie preferentemente de llanura (14) y a pesar de su resistencia a bajas temperaturas (15), es evidente que no soporta las condiciones climáticas y edáficas imperantes en el sitio de plantación.

La plantación de algarrobos blanco y negro, por su parte, no arrojó resultados satisfactorios para ninguna de las dos especies. Si bien algarrobo blanco alcanzó un $78 \%$ de sobrevivencia en ladera baja, ni éste ni algarrobo negro superaron el $35 \%$ en ladera alta. La mayor sobrevivencia del primero no se correspondió con el crecimiento en altura, que resultó muy bajo para la especie. A la inversa, algarrobo negro, del cual subsistieron escasos ejemplares en ladera baja, sobrepasó al resto de las especies en crecimiento en altura, en la exposición Este, no obstante, el incremento fue apenas de 11,2 $\mathrm{cm}$ en 2,5 años. Ambas especies prosperan principalmente en el fondo de los valles o en las lomadas bajas, asociadas con suelos bastante profundos y con la humedad de los cursos de agua. A pesar de la cercanía geográfica con su área de distribución natural, las condiciones de suelos someros, bajas temperaturas y fuertes vientos, imperantes en la zona del ensayo, fueron desfavorables para su establecimiento. Estos resultados confirman anteriores experiencias realizadas por el equipo de trabajo en la región.

La época de plantación fue determinante para la sobrevivencia y el crecimiento en altura del eje principal de las plantas durante los primeros meses, resultando más favorable la plantación de primavera que la de otoño. Mientras el déficit hídrico es un factor muy importante para el establecimiento de coníferas en la zona (25), las temperaturas mínimas parecen serlo para las especies nativas. Así, las coníferas pueden superar inviernos rigurosos si cuentan con una adecuada reserva de agua en el suelo, mientras que las especies probadas en este ensayo sufrieron las consecuencias de las bajas temperaturas invernales. Prueba de ello son los daños observados a la salida del invierno en hojas y tallos. Muchas plantas rebrotaban con la llegada de la primavera, pero volvían a sufrir daños al invierno siguiente.

Una alternativa válida, al menos para las especies que mostraron mejor sobrevivencia y creci- 
miento, sería la utilización de plantines de mayor edad y tamaño (12-24 meses-0,50/0,80 m), plantados a mediados de la primavera, luego de una lluvia abundante. Con esto se esperaría un mayor tamaño inicial de las plantas, mayor rusticidad, y, por tanto, mejor tolerancia a heladas, la que está directamente relacionada con la edad del plantín y su rusticidad $(18,19)$. Ensayos con plantines de Prosopis juliflora de 45 a $120 \mathrm{~cm}$ se llevaron a cabo con éxito en la India (Luna 1996, citado por Pasiecznik) (26), pero es muy importante proveerles un abundante pan de tierra para el desarrollo equilibrado de los sistemas aéreo y radicular (27).

En cuanto al efecto de la exposición, los resultados fueron disímiles según la variable analizada. La sobrevivencia fue, en líneas generales, mayor en la exposición Norte, para todas las especies, tanto en ladera baja como alta, salvo para el molle, que en ladera baja tuvo mayor sobrevivencia en las exposiciones Oeste y Este. En términos de crecimiento en altura del eje principal, la exposición que se diferenció del resto fue la Sur, pero sólo por el desempeño del molle en ladera alta. La aparente discrepancia en el comportamiento de las variables se explica si se considera la susceptibilidad de estas especies a las bajas temperaturas. Es posible que las especies sobrevivan más en la exposición Norte por su mayor temperatura invernal, a pesar de que esta exposición exhibe un déficit hídrico más pronunciado $(18,19)$. La menor sobrevivencia en exposición Sur se ve compensada, en el caso del molle, por un mayor crecimiento en altura, favorecido por condiciones más frescas y sombreadas que reducen la evapotranspiración, preferidas por la especie $(1,3)$.

Los resultados indican que las especies crecen más en ladera alta que baja. Si bien éste es sólo un análisis exploratorio para detectar tendencias, efectuado a los pocos meses de la plantación, esta diferencia a favor de la ladera alta podría atribuirse a mayores temperaturas, tanto de invierno como de verano, ya que la ladera baja actuaría como depósito de masas de aire frío que se acumulan, por su mayor peso, en estas zonas.

Por otra parte, el efecto de la cobertura de la vegetación acompañante sobre la sobrevivencia y la condición general de los plantines fue significativo. Las mayores tasas de sobrevivencia y las plantas de mejor condición general se produjeron bajo la cobertura de la vegetación herbácea acompa- ñante. Esta respuesta puede deberse, principalmente, a la protección que proporcionan los pastos altos contra las variaciones extremas de temperatura $\mathrm{y}$, en particular, las bajas temperaturas del invierno a las que las especies estudiadas resultaron muy sensibles. Si bien el grueso de la bibliografía hace referencia al efecto benéfico de ciertos arbustos sobre la germinación y el crecimiento de algunas especies en zonas áridas y semiáridas (28, 21), los mismos principios de modificación de microhábitats serían de aplicación para los pastizales de altura donde se instaló el presente ensayo. Así, los individuos que se implanten bajo la cobertura de los pastos, o en su área de influencia, tendrían mayores probabilidades de sobrevivir a las condiciones climáticas rigurosas en las mayores alturas de las Sierras de Córdoba.

Ya en 1957, Norman et al., citado por Geiger (29), describieron los efectos diferenciales de pastos altos y bajos sobre las temperaturas mínimas. En su estudio, el número de noches con heladas en zonas con pastos muy bajos $(2-3 \mathrm{~cm})$ fue el doble que en zonas con pastos altos $(30-45 \mathrm{~cm}$ de altura), demostrando una relación directa entre el tamaño de la vegetación herbácea y la moderación del microclima subyacente. Es posible, además, que en el presente estudio los plantines protegidos bajo las grandes matas de Stipa y Festuca sufrieran en menor medida los efectos de los fuertes vientos (29).

\section{CONCLUSIONES}

El establecimiento de algunas especies valiosas del Chaco occidental en áreas de pastizales de altura resulta sumamente dificultoso. Las condiciones ambientales de este piso fitogeográfico se presentan muy desventajosas para las especies estudiadas, lo que queda de manifiesto en la baja sobrevivencia y la reducción en la altura promedio al cabo de varios años de plantación, con la sola excepción del molle.

Teniendo en cuenta estas limitantes puede aseverarse que la mejor época de plantación es la primavera y las exposiciones más propicias para el arraigo de las plantas son la Norte y Oeste, según se plante en ladera alta o baja respectivamente. En cambio, el mayor crecimiento promedio en altura se produjo en molle, en la exposición 
Sur. Las plantas presentaron mejor sobrevivencia y estado general bajo la protección de los pastos acompañantes, o en el borde de los mismos.

Molle es la única especie que creció significativamente, pero sólo en exposición Sur. Por lo tanto se la podría señalar como una especie adecuada para forestaciones en pastizales degradados hasta 1.250 m s.n.m., con preferencia en las laderas más frescas y húmedas de las Sierras de Córdoba.

\section{AGRADECIMIENTOS}

Las autoras agradecen a Patricia Ingaramo, por su participación durante las primeras etapas del proyecto; a Atilio Palacios, encargado de la Reserva Natural Vaquerías, por su colaboración, a Nieves Heredia y familia, por su participación en las actividades de plantación y cuidados culturales, a Mónica Sagadín y Silvia Palacios que dieron su apoyo para las mediciones de campo y posterior procesamiento de los datos, a la Asociación Civil Los Algarrobos, por su valiosa gestión interinstitucional y a los estudiantes de Cs. Agropecuarias y Cs. Biológicas que colaboraron con la plantación y mediciones del ensayo.

\section{BIBLIOGRAFIA}

(1) LUTI, R., M. BERTRAN DE SOLIS, F. M. GALERA, N. MÜLLER DE FERREIRA, M. BERZAL, M. NORES, M. HERRERA, J. C. BARRERA. Vegetación. En: MIATELLO, R. Geografía física de la provincia de Córdoba, Bs. As.: Ed. Boldt, 1979, p. 297-367.

(2) CASTILLO SANCHEZ, V. Ensayo y evaluación de técnicas de repoblación con pino carrasco (Pinus halepensis Mill) en ambientes mediterráneos semiáridos. En: VALDEBENITO, R. G. y BENEDITTI, R. S. Forestación y silvicultura en zonas áridas y semiáridas de Chile. Stgo., Chile. FONSIP-CORFO,1997, p. 34-43.

(3) MARTIJENA, N. Estructura de Lithraea ternifolia en la cuenca del arroyo Vaquerías. FCEFyN, UNC. Seminarios I y II de la carrera de Cs. Biológicas. 1980, 56 p

(4) BERTRAN DE SOLIS, M. A. Estructura y composición de la vegetación. Informe de Síntesis del proyecto $\mathrm{Pa}$ chón-Achala del Programa MAB6, período 1978-80, 1981, $64 \mathrm{p}$.

(5) ESTRABOU, C. Relevamiento cartográfico de variante fisonómicas de la cuenca. La Quebrada. FCEFyN, UNC. Seminario I y II de la carrera de Ciencias Biológicas. Córdoba, 1983, $49 \mathrm{p}$

(6) KEEGAN, J. Crecimiento individual, estructura y dinámica poblacional de Fagara coco (Gill.) Engler, en un área perturbada de las Sierras Chicas de Córdoba. FCEFyN, UNC. Seminario de la carrera de Ciencias Biológicas, 1984, $67 \mathrm{p}$
(7) JOSEAU, M. J., P. INGARAMO, G. VERZINO, M. DORADO, J. L. ALVAREZ. Vivero Forestal Educativo. II Jornada de Extensión, 1999, 28 p

(8) VERZINO; G., J. JOSEAU, M. DORADO, P. INGARAMO, M. DIAZ. Reforestación experimental con especies nativas en cuencas de montaña degradadas de las Sierras Chicas, Córdoba. Cosecha de frutos y producción de plantines. II Jornada de Extensión, F.C.A, U.N.C., Córdoba 1999, p. 56.

(9) VERZINO, G., S. DE MARINIS, J. JOSEAU. Especies del Chaco Serrano. Fagara coco (Gill.) Engler. Estudios preliminares de domesticación. VII Jornadas de Investigación en Ciencias Agropecuarias. F.C.A, U.N.C., Córdoba, 1999, p. 199.

(10) VERZINO, G., P. WILLIAMS, M. SAGADIN, M. JOSEAU. Multipurpose tree species for sustainable development in Valle de Punilla, Córdoba, Argentina. Women in Natural Resources (U.S.A.), 1995, Vol. 17, $\mathrm{N}^{\circ} 2$, p. $28-31$.

(11) LARA RICO, R. Schinopsis haenkeana. En: FAO. Especies arbóreas y arbustivas para las zonas áridas y semiáridas de América Latina. Serie: Zonas Aridas y Semiáridas $\mathrm{N}^{\circ}$ 12. Santiago, Chile: FAO, 1997, p. 93-97.

(12) KARLIN, U. O., R. COIRINI, L. CATALAN, R. ZAPATA. Argentina. En: FAO. Especies arbóreas y arbustivas para las zonas áridas y semiáridas de América Latina. Serie: Zonas Aridas y Semiáridas $N^{\circ} 12$, Santiago, Chile: FAO, 1997. p. 3-71.

(13) VERGA, A. Algarrobos como especies para forestación: Una estrategia de mejoramiento (Segunda nota). SAGPyA Forestal (Buenos Aires), 2000, Vol. 17, p. 2-9.

(14) BILONI J. S. 1990. Arboles autóctonos argentinos. Bs. As.: Tipográfica Ed. Arg. 335 p.

(15) CESERE, M. S., A. MEEHAN, M. BOETTO. Plantas nativas. Su uso en espacios verdes urbanos. Argentina: Ed. Eudecor, 1997, 95 p.

(16) IZURIETA, G. Los árboles de Córdoba. Córdoba: Secretaría de Agricultura, Ganadería y Recursos Renovables, 1998, $108 \mathrm{p}$

(17) BRACK EGG, A. Plantas nativas utilizadas en el Perú en agroforestería. Bosques y Desarrollo, 1993, Año 4, $\mathrm{N}^{\circ} 8$, p. 22-34.

(18) SPURR, S., B. BARNES. Ecología Forestal. México: Ed. AGT. 1982. 690 p.

(19) DONOSO Z., C. Ecología forestal. El bosque y su medio ambiente. $5^{\text {a }}$ ed., Stgo. de Chile: Universitaria S.A., 1981, $368 \mathrm{p}$.

(20) BARCHUK, A. H., M. P. DIAZ, F. CASANOVES, M. G. BALZARINI, U.O. KARLIN. Experimental study on survival rates in two arboreal species from the Argentinean Dry Chaco. Forest Ecology and Management, 1998, Vol. 103, p. 203-210.

(21) BARCHUK, A. H., C. CARRANZA. Plantas nodrizas en el establecimiento de Prosopis flexuosa en el Chaco Arido. Reunión Nacional del Algarrobo y III Reunión de la AAPro, Mendoza. 2000 , p. 54

(22) MC CULLAGH, P., J. NELDER. Generalized Linear Models. London: Chapman \& Hall. 1989. 521 p.

(23) FRANCIS, B., M. GREEN, C. PAYNE. The GLIM System. Release 4 Manual. Oxford: Clarendon Press, 1994, $871 \mathrm{p}$.

(24) SERRA, M. T. Schinus molle. En: FAO. Especies arbóreas y arbustivas para las zonas áridas y semiáridas de América Latina. Serie: Zonas Aridas y Semiáridas $\mathrm{N}^{\circ} 12$ Santiago, Chile: FAO, 1997, p. 237-243.

(25) RAVELO, A., P. INGARAMO, R. ZANVETOR, G. VERZINO. Evaluación agroclimática para la identifica- 
BOSQUE 25(1): 53-67, 2004 Comportamiento inicial de especies nativas del Chaco Occidental en plantaciones en zonas de pastizales de altura...

ción de los orígenes más promisorios de Pinus elliottii para el Valle de Calamuchita, Cba. II Congr. Ftal. Arg. y Latinoamericano. En: Bosque Cultivado. Misiones. 1997. p. $175-178$

(26) PASIECZNIK, N. M. The Prosopis juliflora-Prosopis pallida Complex: A monograph. Coventry, U.K.: HDRA. 2001. 162 p.

(27) PASIECZNIK, N. M. Prosopis provenance research in Cape Verde. In: J. C. TEWARI, N. M. PASIECZNIK, L. N. HARSH, P. J. C. HARRIS. Prosopis species in the Arid and Semiarid Zones of India. Coventry, U.K 1998. p.73-77.

(28) VALIENTE-BANUET, A., E. EZCURRA. Shade as a cause of the association between the cactus Neobuxbaumia tetetzo and the nurse plant Mimosa luisana in the Tehuacán Valley, México. Journal of Ecology, 1991, Vol. 79, p. 961-971.

(29) GEIGER, R. The climate near the ground. Revised edition. Cambridge, Massachusetts: Harvard University Press, 1971. $611 \mathrm{p}$. 